\title{
Editorial
}

\section{RELATO INTEGRADO E A DIVULGAÇÃO CORPORATIVA PARA A SUSTENTABILIDADE}

\author{
Elise Soerger Zaro \\ E-mail: elise_sz@yahoo.com.br \\ Doutora em Controladoria e Contabilidade pela Universidade de São Paulo (USP) \\ Professora da Faculdade de Administração, Ciências Contábeis e Economia da Universidade Federal da Grande Dourados
}

\section{INTRODUÇÃO}

As empresas têm sofrido muita pressão de grupos de partes interessadas para que demonstrem responsabilidade corporativa, pelo menos, desde a década de 1970. Com base na definição de sustentabilidade do Triple Bottom Line, as entidades devem se preocupar com seus impactos e resultados sociais, ambientais e econômicos (Gray et al., 1996). Não é possível apenas que se preocupe com a maximização da riqueza dos sócios, a empresa normalmente utiliza mão de obra, recursos e causa impactos na comunidade, portanto, tem a responsabilidade de buscar impactos positivos à sociedade e ao meio ambiente (Moser \& Martin, 2012). Diante dessa discussão, as organizações foram pressionadas a reportar suas ações e impactos de sustentabilidade. Essas divulgações passaram por diversos formatos, sempre com o direcionamento de apresentar transparência sobre suas atividades, seus riscos e resultados. No decorrer do tempo, houve discussões tanto no ambiente empresarial, quando no ambiente acadêmico sobre a qualidade das informações não financeiras apresentadas pelas entidades e sobre como aumentá-la (Moser \& Martin, 2012).

Muitas iniciativas surgiram com o intuito de auxiliar as empresas a realizar as divulgações de responsabilidade corporativa de maneira mais adequada. Uma delas é o relato integrado $<$ RI $>$. A proposta do relato integrado é incentivar as entidades a adotar um processo de relatar que considere a conectividade e a interdependência de fatores financeiros e não financeiros, para demonstrar como a empresa gera valor. Não se limita à divulgação de um documento específico, mas uma adequação de todo o processo de produção da informação, que deve refletir como a gestão enxerga a empresa (International Integrated Reporting Council IIRC, 2013).

O Relato Integrado tem ganhado visibilidade no mundo corporativo e nesse momento ganha especial atenção com o anúncio da intenção de fusão do The International Integrated Reporting Council e Sustainability Accounting Standard Board (SASB) para a criação do Value Reporting Foundation, realizado em dezembro de 2020. Adicionalmente, em 2021, com a comemoração dos 10 anos da criação do The International Integrated Reporting Council e com a divulgação da primeira revisão da Estrutura Conceitual Internacional para Relato Integrado, em 19 de janeiro de 2021. Ainda no cenário internacional, o Relato Integrado tem um papel protagonista na discussão promovida pela IFRS Foundation sobre normas internacionais de divulgação sobre sustentabilidade. No Brasil, a CVM aprovou pela Resolução CVM 14 a Orientação Técnica CPC 09 - Relato Integrado e ainda o relato integrado ganhou visibilidade por sua obrigatoriedade de divulgação pelos órgãos públicos federais.

O principal conceito da proposta de relato integrado é o pensamento integrado, ou seja, que a tomada de decisão de toda a organização, nos mais diversos níveis, considere todos os aspectos e impactos relevantes da decisão. Para isso, foi proposto que sejam consideradas seis dimensões, chamadas de capitais. Os capitais são: financeiro; manufaturado; social e de relacionamento; humano; intelectual e natural (IIRC, 2013). Assim, a gestão e todos os demais atores são incentivados a refletir sobre a criação de valor de uma maneira holística, identificando quais são os fatores materiais para cada organização. Cada modelo de negócios tem as suas características especificas, então os tomadores de decisão e preparadores das informações devem definir se as seis dimensões propostas devem ser mantidas ou se realizam alguma alteração, podendo acrescentar, modificar ou retirar qualquer item de acordo com a capacidade daquele aspecto ter impacto na decisão, principalmente dos provedores de capital (Baboukardos \& Rimmel, 2016).

Uma das principais críticas que o relato integrado enfrenta refere-se à escolha de direcionar as informações às necessidades dos provedores de capital financeiro. A iniciativa pretende melhorar a qualidade das informações corporativas, para demonstrar de forma abrangente e multidimensional como a empresa opera e quais resultados ela gera. Isso pode atender às necessidades informacionais de vários stakeholders, ou seja, várias partes relacionadas à empresa. Por outro lado, tendo como ponto de partida as necessidades informacionais dos provedores de capital financeiro, o relato integrado tende a apresentar um viés. Sendo assim, deixar de reportar aspectos considerados relevantes para outros stakeholders, mas não para os financiadores da organização. 
O principal documento que norteia a aplicação do Relato integrado é a estrutura conceitual internacional para o relato integrado publicada pelo The International Integrated Reporting Council - IIRC. Esse framework é essencialmente principiológico, seguindo as tendências das normas internacionais de contabilidade. Apresenta os princípios fundamentais para a elaboração de um relato integrado, sendo flexível o suficiente para que todas as organizações possam adotar, independente do tamanho, se tem fins lucrativos ou outra particularidade (IIRC, 2013). O framework estabelece também os elementos de conteúdo mínimos que devem ser apresentados pela organização para que ela possa declarar que está reportando de acordo com o relato integrado. Adicionalmente, alguns outros aspectos que não são obrigatórios, mas encorajados para reporte (IIRC, 2013).

\section{PRINCÍPIOS DO RELATO INTEGRADO E DISCUSSÕES LEVANTADAS PELA LITERATURA}

A proposta de relato integrado baseada em princípios visa promover uma mudança substancial na gestão e no processo de preparação das informações, o primeiro deles trata-se de foco estratégico e orientação para o futuro, ou seja, a definição da estratégia da organização deve ser focada na conectividade e pautada pelo pensamento integrado.

Um dos pontos principais do relato integrado é implantar o pensamento de longo prazo, estabelecendo metas e projetos para o curto, médio e longo prazo (IIRC, 2013). Assim, a empresa pode ponderar riscos e oportunidades pensando em sua continuidade, ou seja, sua sustentabilidade. No entanto, em uma empresa com fins lucrativos existem vários interesses influenciando as decisões, entre eles o resultado de curto prazo que na maioria das vezes será base para remuneração de gestores e acionistas e a capacidade de geração de caixa que influencia a capacidade de pagamento de fornecedores e credores. Apesar da maioria desses grupos se beneficiar de um planejamento que dê maior solidez no longo prazo, em muitos casos é necessário um sacrifício de curto prazo para aumentar essa solidez. Assim, surge um fenômeno da psicologia interessante para ser analisado, que se refere ao comportamento compulsivo e de autocontrole que vão determinar as decisões em privilegiar os resultados de curto prazo ou de longo prazo, respectivamente.

Os normatizadores e gestores públicos precisam pensar nos mecanismos para incentivar um comportamento coletivo de autocontrole e planejamento. Em relação aos investidores, são necessárias ferramentas para alterar o comportamento de curto prazo, com característica de especulação e passar a uma base de investidores que acredita nos resultados da empresa e faz investimentos de longo prazo (Serafeim, 2015). Existem algumas alternativas adotadas ao redor do mundo para buscar essa alteração de base de acionistas que vai de melhorar a educação financeira até a criação de classes diferenciadas de ações. Os gestores também podem ser incentivados a estender o horizonte temporal com um desenho adequado do plano de remuneração.

Os incentivos devem compreender a busca pelo autocontrole, levando os tomadores de decisão a adotarem um pensamento de longo prazo e também a preocupação com múltiplos fatores. A sustentabilidade financeira e não financeira deveria ser avaliada considerando a conectividade entre os fatores que afetam a capacidade da organização de gerar valor: por exemplo, em uma universidade privada as bolsas de estudo oferecidas com recursos próprios podem significar abrir mão de entrada de caixa, o que do ponto de vista financeiro isoladamente é ruim. Por outro lado, se você analisar a geração de capital intelectual que esses indivíduos têm, ao se envolver com projetos, a capacitação que eles recebem e o impacto geral na sociedade no período de assistência e posteriormente, pode-se perceber a geração de capital intelectual, humano e social e de relacionamento. Esse exemplo busca demonstrar a necessidade de analisar o processo de criação de valor como um todo, considerando suas interdependências. Pois as empresas são ambientes complexos e é necessário ter uma consideração ativa sobre todos os aspectos relevantes para compreender seus impactos e resultados.

Existem críticas que talvez o relatório fique muito complexo e as informações não sejam usadas pelos usuários (Bucaro et al., 2017). Tendo em vista a proposta de demonstrar o processo de criação de valor da empresa considerando múltiplos capitais, existem pesquisadores ponderando que a divulgação pode ficar muito complexa e que os usuários não consigam extrair as informações que necessitam. Como contraponto dessa crítica, existe a orientação de que as empresas adequem a linguagem e utilizem recursos gráficos para facilitar o entendimento das informações apresentadas.

Dentre os princípios mais discutidos pelo relato integrado está o princípio da materialidade, ele foi o primeiro a ser discutido pelo Corporate Reporting Dialogue, uma coalizão formada pelo IIRC, Carbon Disclosure Project CDP, CDSB, FASB, GRI, IFRS, ISO e SASB para harmonizar os conceitos de diversas iniciativas de divulgação corporativa, visando criar sinergia entre essas organizações. Refere-se principalmente à definição dos temas que são mais sensíveis à organização, estabelecendo as prioridades na tomada de decisão e posteriormente no reporte. Segundo a discussão do Corporate Reporting Dialogue (2015) a informação material é aquela que tem razoável capacidade de fazer diferença na análise em questão. Essa é uma definição abrangente que pode se encaixar no contexto de todas as instituições envolvidas. No entanto, o exercício de definir quais são os temais mais relevantes para a administração, para a tomada de decisão e posteriormente para a divulgação, não é trivial e não é fácil. 
A definição dos temas materiais é um dos pontos mais críticos para a qualidade do planejamento, avaliação e reporte, por definir quais são os itens que receberão maior atenção, maior recurso ou maior necessidade de transparência por ordem de prioridade. O princípio da materialidade precisa ser analisado em conjunto com o princípio da concisão. Ao mesmo tempo que é necessário analisar e divulgar todos os fatores e aspectos relevantes para a organização que terão a capacidade de influenciar as decisões, também é importante analisar qual a forma mais simples e clara de passar a informação. Outro aspecto refere-se a não apresentar informações que não sejam materiais, pois isso tira o foco dos temas que realmente precisam ser analisados.

Existem algumas técnicas de definição de materialidade que foram desenvolvidas para definir quais são os temas prioritários das organizações, como a matriz de materialidade consultando as partes interessadas, a consulta ao público interno à organização, ou a definição por parte da gestão sobre os temas materiais a serem abordados nos relatórios. Muitas empresas descrevem o processo de definição de materialidade para dar maior credibilidade às informações (Fasan \& Mio, 2016).

Cabe destacar que os normatizadores da área contábil também estão debatendo sobre a redução das informações divulgadas por dar maior foco apenas às informações relevantes (Financial Accounting Standards Board, 2015).

A atenção aos princípios de concisão e materialidade são importantes, devido a problemas que as pessoas enfrentam quando existe excesso de informação. Como destacados por Bazerman e Moore (2009), as pessoas aplicam filtros nas informações, porém muitas vezes isso ocorre inconscientemente e automaticamente, o que as leva a ignorar ou negligenciar informações. Adicionalmente, Hirshleifer e Teoh (2003) demonstram evidências de que os investidores possuem atenção e poder de processamento limitado, consequentemente, informações apresentadas de forma mais saliente e de uma forma clara são mais bem absorvidas pelos investidores.

\section{RELATO INTEGRADO, VISIBILIDADE NO MERCADO E BENEFICIOS ECONÔMICOS}

O órgão que desenvolveu a estrutura internacional para o relato integrado é o International Integrated Reporting Council - IIRC. Sua criação foi em 2011, idealizada pelo Accounting for Sustainability A4S, organização criada pelo Príncipe de Gales para auxiliar as organizações a utilizarem a contabilidade e a divulgação corporativa para buscar uma atuação mais sustentável. Em 2013, a primeira estrutura conceitual foi divulgada para orientar as organizações em como se engajar com o relato integrado. Como pode-se perceber, em 2021 essa organização completa 10 anos, mostrando uma sobrevida da proposta.

Um dos aspectos que podemos levar em consideração para verificar que a iniciativa tem ganhado atenção, em dezembro/2021, o International Integrated Reporting Council - IIRC e o Sustainability Accounting Standard Board (SASB) anunciaram a intenção de realizar uma fusão entre as duas instituições e criar o Value Reporting Foundation. A proposta é aliar a base principiológica do relato integrado com o arcabouço de normatização do SASB, podendo contribuir para melhorar a divulgação corporativa no mundo. Conforme salientando pelo IIRC (2021a), existem cerca de 2.500 organizações de 70 países realizando a estrutura conceitual para relato integrado em suas divulgações corporativas.

Outro fato relevante que aconteceu com a celebração dos 10 anos da criação do International Integrated Reporting Council IIRC, foi a divulgação da versão revisada da Estrutura Conceitual Internacional para Relato Integrado. Ela foi divulgado em janeiro de 2021 em evento online (International Integrated Reporting Council IIRC, 2021b) buscando o desenvolvimento de informações mais úteis para a tomada de decisão (International Integrated Reporting Council IIRC, 2021a).

Além disso, a organização The IFRS Foundation, ligada ao IASB, que são responsáveis pela divulgação das normas internacionais de contabilidade, divulgaram um artigo de consulta intitulado "Sustainability reporting - demand for global sustainability standards", que pode ser traduzido como "Divulgação de Sustentabilidade - Demanda por normas globais de sustentabilidade" (tradução nossa). Essa consulta sinaliza uma demanda do mercado financeiro por informações de sustentabilidade com padrões de qualidade, credibilidade e comparabilidade. $O$ CEO do IIRC anunciou no IFRS Foundation announcement que estão trabalhando em conjunto com o IFRS Foundation para que essa normatização incorpore as experiências do relato integrado.

Um objeto de pesquisa interessante refere-se a analisar o lobbying no processo de construção da versão revisada da Estrutura Conceitual Internacional para Relato Integrado e também no processo de normatização das divulgações sobre sustentabilidade promovidas por órgãos reguladores como o IASB/IFRS Foundation ou FASB.

Em concordância com o anúncio da IFRS Foundation para a necessidade de uma normatização internacional sobre a divulgação de sustentabilidade, de acordo uma pesquisa realizada pela $\mathrm{PwC}$ (2014), os investidores expressaram suas preferências sobre quais informações buscam em um relatório corporativo e a forma de apresentação das mesmas. A partir de entrevistas com 85 profissionais de investimento ( $49 \%$ sell side, $42 \%$ buy side e $9 \%$ de agências de rating) identificou-se, entre outros achados, que os participantes esperam encontrar informações claras sobre como os resultados financeiros se relacionam com o modelo de negócios, os riscos identificados e as estratégias da companhia e melhorar os links entre os relatórios das organizações, para facilitar a análise da performance da companhia. 
O relato integrado é uma das alternativas para atender as necessidades apontadas pelos investidores, sendo que propõe demonstrar o processo de criação de valor englobando todos os fatores afetados pelas atividades da organização, demonstrando a conectividade entre as informações e a uniformização do discurso entre todos os reportes da empresa, pois os preparadores das diversas áreas da organização estarão em contato.

A divulgação corporativa de acordo com a estrutura conceitual para relato integrado, deve divulgar de forma concisa todos os fatores que influenciam o desempenho da organização no curto, médio e longo prazo. Espera-se que, assim, essas informações sejam capazes de auxiliar os provedores de capital a aumentar a acurácia de suas previsões em relação ao desempenho futuro do empreendimento e melhorar a alocação de recursos (Cheng et al., 2015).

Os benefícios decorrentes das divulgações integradas pela organização podem apresentar diferenças de intensidade de acordo com o horizonte do investidor, curto ou longo prazo. Segundo Serafeim (2015), investidores de longo prazo têm maior probabilidade de manter ações de empresas que divulgam informações das perspectivas de longo prazo, uma vez que levam essas informações em consideração para tomada de decisão, previsão do valor da empresa e monitoramento da administração, ou seja, os investidores com orientação de longo prazo utilizam mais as informações contidas no relato integrado.

O principal objetivo do relato integrado <RI $>$ é aumentar a qualidade da informação disponível para os provedores de capital financeiro, diminuindo a assimetria de informação e resultando na alocação mais eficiente do capital (Cheng et al., 2014, IIRC, 2013). A assimetria de informação ocorre quando uma das partes da transação tem vantagem de informação em relação a outra, esse problema pode ser dividido em dois tipos: seleção adversa e risco moral. A seleção adversa é o tipo de assimetria de informação que uma das partes da transação, por exemplo a administração ou investidores majoritários, possuem mais informação sobre as condições correntes e futuras da organização do que os demais stakeholders e essas informações podem ser utilizadas de forma a reduzir a habilidade do usuário na tomada de decisão. O risco moral refere-se à diferença de informação em relação às partes da transação sobre o efetivo comportamento, em especial da gestão, e impossibilidade de verificação se suas ações estão alinhadas aos interesses do principal após uma transação (Scott, 2012, p. 21).

Alguns estudos já desenvolvidos buscam entender qual a relação da adoção do relato integrado com a redução da assimetria de informação e a reação do mercado em relação a essas mudanças na forma de preparação das informações. Barth et al. (2017) destacam que o valor da empresa aumenta com a adoção do relato integrado no contexto da África do Sul. Baboukardos \& Rimmel (2016) demonstram um aumento da relevância das informações entre as empresas que divulgam relato integrado, também olhando para o contexto institucional da África do Sul. São apresentadas evidências do aumento da acurácia dos analistas após a disponibilização dessas informações (Bernardi \& Stark, 2018; Flores et al., 2019). Também foram identificadas evidências da redução do custo de capital das empresas que adotam relato integrado (García-Sánchez \& Noguera-Gámez, 2017; Zaro, 2019).

\section{RELATO INTEGRADO NO SETOR PÚBLICO}

A comunicação deve ser concisa para que o usuário tenha capacidade de acessar uma visão holística, incluindo as estratégias, a governança, a performance e as perspectivas da empresa (IIRC, 2013). A proposta para relato integrado tem seu foco principal em corporações, no entanto, sugere-se que a estrutura conceitual pode ser aplicável a outros setores e tipos de organizações, especialmente aqueles com foco mais amplo do que gerar lucro, tais como governos e organizações sem fins lucrativos (Simnett \& Huggins, 2015).

Em outubro/2019, o Brasil foi pioneiro em tornar obrigatória a adoção do relato integrado por todos os órgãos públicos federais, através da Decisão Normativa - TCU N ${ }^{\circ} 178$. O relato integrado é uma iniciativa que surgiu com o intuito de orientar e encorajar as organizações a realizar divulgações integrando fatores financeiros e não financeiros. O principal objetivo do relato integrado é demonstrar o processo de criação de valor em uma perspectiva de longo prazo.

Considerando que a administração pública tem por essência gerar resultados em múltiplas perspectivas e não somente financeiro, o relato integrado demonstra um potencial de auxiliar os envolvidos na administração, os preparadores das informações e o público externo a entenderem melhor os resultados, os impactos e os valores gerados considerando os capitais financeiro, manufaturado, intelectual, humano, social e de relacionamento e natural.

Diante desse cenário, surge a discussão sobre as vantagens e desvantagens da adoção mandatória comparativamente com a adoção voluntária. A divulgação de informações relativas à Responsabilidade Social Corporativa (RSC) iniciou de forma voluntária e existem poucas normas que estabelecem especificamente as divulgações de sustentabilidade que devem ser realizadas pelas organizações (Nikolaeva \& Bicho, 2011). Várias iniciativas voluntárias surgiram desde então.

A literatura aponta que as organizações que se envolvem voluntariamente tendem a ter um efeito maior na qualidade do disclosure e nos benefícios percebidos após a adoção (Ahmed et al., 2013). Isso pode ser resultado de melhores práticas, devido ao envolvimento por iniciativa própria, pela sinalização para o mercado de que a 
corporação tem melhores práticas por estar se engajando voluntariamente à iniciativa, ou outros benefícios percebidos pela entidade. Por outro lado, a adoção voluntária tende a não seguir um padrão, tem o potencial de apresentar vieses como a apresentação de mais informações positivas do que negativas e reduzida comparabilidade.

A obrigatoriedade na adoção pode trazer benefícios na credibilidade e comparabilidade das informações, inclusive influenciando a adoção de outras práticas voluntárias (Einhorn, 2005), porém, pode enfrentar resistência dos preparadores a adotar uma iniciativa que eles não estão preparados ou engajados para a aplicação e reduzir a qualidade das informações prestadas.

A África do Sul foi pioneira na obrigatoriedade da adoção do relato integrado para as empresas de capital aberto que negociavam ações na Bolsa de Valores de Johanesburgo, desde 2010 (Baboukardos \& Rimmel, 2016; Barth et al., 2017). Comparativamente com resultados de estudos da adoção voluntária do relato integrado, pudemos perceber algumas diferenças, como por exemplo a redução do custo de capital não foi observado nas empresas da África do Sul, mas foi observado entre as empresas que adotaram voluntariamente (García-Sánchez \& Noguera-Gámez, 2017; Zaro, 2019).

O Brasil foi pioneiro na adoção mandatória do relato integrado para as instituições públicas federais. Em uma análise preliminar de 12 universidades públicas federais, escolhidas aleatoriamente, identificou-se uma redução média de 49\% no número de páginas do relatório de 2017 a 2019, o que indica que as universidades estão buscando aplicar o princípio de materialidade e concisão nos relatórios.

Uma análise não sistemática, apenas pela comparação visual desses relatórios, indica que as universidades estão utilizando mais recursos gráficos para tentar deixar seus resultados mais claros e informativos, o que pode ser um facilitador na transparência das contas públicas, caso seja usado adequadamente.

\section{O FUTURO A PARTIR DA CONSOLIDAÇÃO DO RELATO INTEGRADO}

O relato integrado é uma iniciativa relativamente recente, se considerarmos que a divulgação da estrutura conceitual para relato integrado aconteceu em 2013. Pelas informações apresentadas anteriormente é possível perceber que ela se difundiu no mundo e está se consolidando, em especial por estabelecer parcerias com as maiores instituições que se preocupam com iniciativas de sustentabilidade corporativa e de divulgação corporativa para a sustentabilidade. Diante desse cenário, apresentam-se alguns exemplos de oportunidades relacionadas ao relato integrado, no âmbito profissional, atuação das empresas e em pesquisa.

Em termos de oportunidade profissional, existe uma vasta gama de tarefas que se relacionam com a elaboração do relato integrado, que vão desde a atuação interna dentro de uma organização, estabelecendo os sistemas de comunicação para transformar o modelo de silos num modelo de fluxos de informação entre os setores para que eles consigam ter uma visão mais holística da organização; à proposição e implementação de ferramentas e controle internos que permitam quantificar, mensurar ou atribuir valor para os mais diversos recursos utilizados ou gerados pelas atividades desempenhadas. Também existe a função de sensibilização e treinamento para difundir o conceito de pensamento integrado por toda a organização. Surgem também figuras muito marcantes nesse mercado que são os consultores e asseguradores. Os trabalhos de consultoria auxiliam as empresas na implementação dos princípios e na institucionalização do relato integrado de uma forma mais célere, o que abre espaço para uma grande diversidade de abordagens. Por fim, ressalta-se a necessidade de asseguração das informações produzidas seguindo os princípios do relato integrado. O que se mostra bastante complexo e uma tarefa que tem uma normatização sendo discutida pelo IAASB, mas ainda sem nenhuma publicação definitiva, sendo um ramo de atuação carente de profissionais capacitados e com uma bagagem multidisciplinar.

Para as empresas, o relato integrado traz a oportunidade de repensar o seu processo de tomada de decisão como um todo, buscando a criação de valor de curto, médio e longo prazo. Do processo de implementação de pensamento integrado, espera-se que resulte em melhora no desempenho de sustentabilidade, redução no impacto ambiental causado por suas atividades, aumento na produção de capital intelectual, melhora no uso dos recursos manufaturados disponíveis, aumento na satisfação dos funcionários por entender o seu papel dentro da organização e pela gestão desse capital específico. Melhor uso dos recursos financeiros e por fim melhores resultados financeiros e ganho em várias dimensões decorrentes da melhor gestão dos seus relacionamentos.

No que tange à pesquisa, já existe um corpo de literatura se formando, porém, é um tópico que ainda oferece muitas oportunidades de pesquisa. Um panorama bastante simplificado das pesquisas no mundo iniciou por investigações exploratórias das vantagens da adoção do relato integrado (Jensen \& Berg, 2012), depois passaram para a investigação dos determinantes da adoção do relato integrado (Fasan \& Mio, 2016; Reitmaier \& Schultze, 2017; Sierra-García et al., 2015), na sequência passaram a analisar atributos da divulgação, fazendo análise dos relatórios (Lai et al., 2016; Melloni, 2015; Melloni et al., 2016)the so-called 'integrated report' (IR. Por fim, a pesquisa evolui para buscar identificar os benefícios econômicos relacionados ao relato integrado (Baboukardos \& Rimmel, 2016; Barth et al., 2017; Bernardi \& Stark, 2018; Flores et al., 2019; García-Sánchez \& Noguera-Gámez, 2017; Zaro, 2019). 
Todos os pontos de pesquisa apresentados anteriormente ainda não têm uma resposta determinística, cabendo outros olhares para essas mesmas questões. Adicionalmente, essa área ainda demanda de pesquisas sobre a qualidade das informações; sobre como os sistemas de remuneração impactam na adoção do relato integrado e na eficiência da implantação do relato integrado. Existem diversos aspectos psicológicos, cognitivos e comportamentais que podem influenciar tanto a adoção, quanto o uso dessas informações que merecem atenção e aprofundamento. Aspectos relacionados a linguagem mais efetiva e mais compreensível também podem ser explorados. Por fim, outros estudos podem ser elaborados para embasar os normatizadores e os gestores públicos nos mecanismos para incentivar um comportamento coletivo de autocontrole e planejamento de longo prazo, sendo necessárias abordagens criativas e ousadas para entender melhor as práticas de relato integrado e como melhorar a sua qualidade e utilidade. 


\section{REFERÊNCIAS}

Ahmed, K., Chalmers, K., \& Khlif, H. (2013). A Meta-analysis of IFRS adoption effects. The International Journal of Accounting, 48, 173-217. https://doi.org/10.1016/j.intacc.2013.04.002

Baboukardos, D., \& Rimmel, G. (2016). Value relevance of accounting information under an integrated reporting approach : A research note. Journal of Accounting and Public Policy, 35, 437-452. https://doi.org/10.1016/j.jaccpubpol.2016.04.004

Barth, M. E., Cahan, S. F., Chen, L., \& Venter, E. R. (2017). The economic consequences associated with integrated report quality: Capital market and real effects. Accounting, Organizations and Society, 62, 43-64. https://doi.org/10.1016/j. aos.2017.08.005

Bazerman, M. H., \& Moore, D. (2009). Judgment in managerial decision making (Issue 7). John Wiley \& Sons, Inc.

Bernardi, C., \& Stark, A. W. (2018). Environmental, social and governance disclosure, integrated reporting, and the accuracy of analyst forecasts. The British Accounting Review, 50(1), 16-31. https://doi.org/10.1016/j.bar.2016.10.001

Bucaro, A., Jackson, K., \& Lill, J. B. (2017). The Influence of CSR Measures on Investorss Judgments When Integrated in a Financial Report Versus Presented in a Separate Report. SSRN Electronic Journal, 1-37. https://doi.org/10.2139/ssrn.2930360

Cheng, M., Green, W., \& Ko, J. C. W. (2015). The Impact of Strategic Relevance and Assurance of Sustainability Indicators on Investors' Decisions. AUDITING: A Journal of Practice \& Theory, 34(1), 131-162. https://doi.org/10.2308/ajpt-50738

Corporate Reporting Dialogue. (2015). Navigating the Corporate Reporting Landscape (p. 21). http://corporatereportingdialogue.com/wp-content/uploads/2015/05/CRD-Mapping-Document_website_070515.pdf

Einhorn, E. (2005). The Nature of the Interaction between Mandatory and Voluntary Disclosures. Journal of Accounting Research, 43(4), 593-621. https://doi.org/10.1111/j.1475-679X.2005.00183.x

Fasan, M., \& Mio, C. (2016). Fostering Stakeholder Engagement: The Role of Materiality Disclosure in Integrated Reporting. Business Strategy and the Environment, 26(3), 288-305. https://doi.org/10.1002/bse.1917

Financial Accounting Standards Board. (2015). Disclosure Framework. http://www.fasb.org/jsp/FASB/FASBContent_C/ ProjectUpdatePage \&cid $=1176156344894$

Flores, E., Fasan, M., Mendes-da-Silva, W., \& Sampaio, J. O. (2019). Integrated reporting and capital markets in an international setting: The role of financial analysts. Business Strategy and the Environment, 28(7), 1465-1480. https://doi.org/10.1002/ bse. 2378

García-Sánchez, I.-M., \& Noguera-Gámez, L. (2017). Integrated information and the cost of capital. International Business Review, 26(5), 959-975. https://doi.org/10.1016/j.ibusrev.2017.03.004

Gray, R., Owen, D., \& Adams, C. (1996). Accounting \& Accountability: Changes and Challenges in Corporate Social and Environmental Reporting. Prentice Hall Europe.

Hirshleifer, D., \& Teoh, S. H. (2003). Limited attention, information disclosure, and financial reporting. Journal of Accounting and Economics, 36(1-3), 337-386. https://doi.org/10.1016/j.jacceco.2003.10.002

International Integrated Reporting Council IIRC. (2013). The International <IR> Framework. http://www.theiirc.org/wp-content/uploads/2013/12/13-12-08-THE-INTERNATIONAL-IR-FRAMEWORK-2-1.pdf

International Integrated Reporting Council IIRC. (2021a). IIRC publishes revisions to International $\langle$ IR $>$ Framework to enable enhanced reporting. https://integratedreporting.org/news/iirc-publishes-revisions-to-international-framework-to-enable-enhanced-reporting/

International Integrated Reporting Council IIRC. (2021b). Our plans for 2021: Globalizing integrated reporting. https:// integratedreporting.org/news/iirc-newsletter-29/

Jensen, J. C., \& Berg, N. (2012). Determinants of Traditional Sustainability Reporting Versus Integrated Reporting. An Institutionalist Approach. Business Strategy and the Environment, 21(November 2011), 299-316. https://doi.org/10.1002/bse.740

Lai, A., Melloni, G., \& Stacchezzini, R. (2016). Corporate Sustainable Development: is 'Integrated Reporting' a Legitimation Strategy? Business Strategy and the Environment, 25(3), 165-177. https://doi.org/10.1002/bse.1863

Melloni, G. (2015). Intellectual capital disclosure in integrated reporting: An impression management analysis. Journal of Intellectual Capital, 16(3), 661-680. https://doi.org/10.1108/JIC-11-2014-0121

Melloni, G., Stacchezzini, R., \& Lai, A. (2016). The tone of business model disclosure: an impression management analysis of the integrated reports. Journal of Management \& Governance, 20(2), 295-320. https://doi.org/10.1007/s10997-015-9319-z

Moser, D. V., \& Martin, P. R. (2012). A Broader Perspective on Corporate Social Responsibility Research in Accounting. The Accounting Review, 87(3), 797-806. https://doi.org/10.2308/accr-10257

Nikolaeva, R., \& Bicho, M. (2011). The role of institutional and reputational factors in the voluntary adoption of corporate social responsibility reporting standards. Journal of the Academy of Marketing Science, 39, 136-157. https://doi.org/10.1007/ s11747-010-0214-5

Reitmaier, C., \& Schultze, W. (2017). Enhanced business reporting: value relevance and determinants of valuation-related disclosures. Journal of Intellectual Capital, 18(4), 832-867. https://doi.org/10.1108/JIC-12-2016-0136

Scott, W. R. (2012). Financial accounting theory (6th ed.). Pearson. 
Serafeim, G. (2015). Integrated Reporting and Investor Clientele. Journal of Applied Corporate Finance, 27(2), 34-51. https://doi.org/10.1111/jacf.12116

Sierra-García, L., Zorio-Grima, A., \& García-Benau, M. a. (2015). Stakeholder Engagement, Corporate Social Responsibility and Integrated Reporting: An Exploratory Study. Corporate Social Responsibility and Environmental Management, 22(5), 286-304. https://doi.org/10.1002/csr.1345

Simnett, R., \& Huggins, A. L. (2015). Integrated reporting and assurance: where can research add value? Sustainability Accounting, Management and Policy Journal, 6(1), 29-53. https://doi.org/10.1108/SAMPJ-09-2014-0053

Zaro, E. S. (2019). Capital and cost of capital and voluntary disclosure of integrated reporting: the role of institutional factors [Universidade de São Paulo]. In Ph.D Dissertation of University Sao Paulo. https://doi.org/10.11606/T.12.2019.tde24052019-121105 